\title{
Changes on the Modulation of the Startle Reflex in Alcohol-Dependent Patients after 12 Weeks of a Cognitive-Behavioral Intervention
}

\author{
Rosa Jurado-Barbac, e, i Gabriel Rubio Valladolid ${ }^{a-d}$ Isabel Martínez-Gras ${ }^{a-d}$ \\ María José Alvarez-Alonsoc Guillermo Ponce Alfaro ${ }^{a-d} \quad$ Alberto Fernández $^{\text {a, } g}$ \\ Stephan Moratti ${ }^{\mathrm{f} g}$ Andreas Heinz ${ }^{\mathrm{h}}$ Miguel Ángel Jimenez-Arriero ${ }^{\mathrm{b}, \mathrm{i}}$ \\ ${ }^{a}$ Department of Psychiatry, School of Medicine, Complutense University of Madrid, b Psychiatry and Mental Health \\ Clinical Management, Hospital 12 de Octubre, ${ }^{C}$ Research Institute Hospital 12 de Octubre (i+12), d Networks for \\ Cooperative Research in Health (RETICS-Addictive Disorders), Carlos III Health Institute, MICINN and FEDER, \\ e Department of Basic Psychology II, School of Psychology, Complutense University, ${ }^{f}$ Department of Basic \\ Psychology I, School of Psychology, Complutense University, and ${ }^{9}$ Center of Biomedical Technology, Laboratory of \\ Cognitive and Computational Neuroscience, Laboratory of Clinical Neuroscience, Technical University of Madrid,

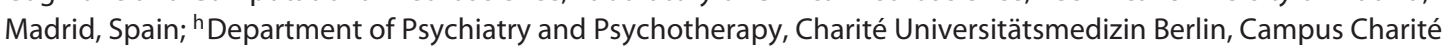

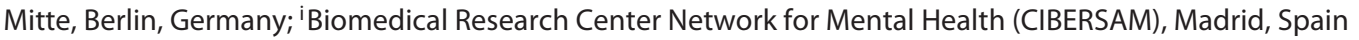

\section{Key Words}

Affective modulation · Alcohol dependence · Cognitive therapy $\cdot$ Follow-up study relapse $\cdot$ Startle reflex

\footnotetext{
Abstract

Aims: Little is known about changes in the modulation of the startle reflex when patients go through an alcohol-dependence treatment in an outpatient facility. In the current study, the affective modulation of the cue-related startle reflex has been used to evaluate changes in the emotional processing of alcohol-related stimuli that occurred after a standard cognitive-behavioral intervention, and to assess the outcome of this intervention. We hypothesized a 'normalization' of the startle inhibition for the alcohol-related cues during the period of treatment. We also assumed that higher startle inhibition at baseline elicited by alcohol cues would predict the relapse on alcohol consumption during treatment. Participants: A total of 98 alcohol-dependent
}

subjects were included who fulfilled DSM-IV criteria for alcohol dependence. A control group of 72 subjects was selected to match demographic characteristics. Measurements: All patients received a standard cognitive-behavioral therapy once a week throughout the study period. Findings: Results show that the startle response differed significantly after 12 weeks of treatment for alcohol-related, neutral and aversive stimuli between alcohol-dependent patients and controls. Low startle responses at baseline to alcohol cues predicted relapse. Conclusions: These results may indicate that the startle reflex is referred to enduring and permanent processes of cue reactivity, and that the emotional processing of alcohol-associated cues assessed with the affect-modulated startle reflex is less altered by interventions attempting to influence explicit cognitions. Furthermore, lower values of the baseline startle reflex elicited by alcohol-associated stimuli were associated with higher probability of relapse on alcohol use.

(c) 2015 S. Karger AG, Basel

\section{KARGER 125}

C 2015 S. Karger AG, Basel

$1022-6877 / 15 / 0214-0195 \$ 39.50 / 0$
Rosa Jurado-Barba

Research Institute Hospital 12 de Octubre (i+12)

Hospital Universitario 12 de Octubre

Avda de Cordoba s/n, ES-28041 Madrid (Spain)

E-Mail rjurado@psi.ucm.es 


\section{Introduction}

It has been demonstrated that alcohol-dependent patients show altered responses to alcohol-related cues [1, 2]. The consumption of a substance in the initial stages of the establishment of an addiction is guided by a powerful positive reinforcement that activates the mesolimbic dopaminergic pathway [3], converting environmental stimuli and received signals into conditioned stimuli that are positively and motivationally marked. The alteration in the reward system as a result of substance use leads to a sensitization of these neural networks. Thus, a reward or incentive memory is established. Incentive-sensitization theories suggest that, once the stimulus-reward association (implicit $\mathrm{S}-\mathrm{S}$ associations) has been established, and due to neural sensitization, addiction is maintained because stimuli are capable of activating the reward and motivational systems even in the absence of pleasure and not because of avoidance of the withdrawal syndrome. This neural sensitization implies an excessive salience attribution to stimuli associated with the substance, which skews attention and behavior, leading to maladaptive compulsive consumption behaviors guided by the reward memory (pathological 'wanting' to take drug) [3, 4].

One of the main goals of rehabilitation programs is the establishment of new learning that will favor the formation of new, more adaptive habits to respond to consumption-related stimuli, for which these stimuli must lose or modulate their incentive value.

The mesolimbic network, specifically the nucleus accumbens, is clearly involved in the attribution of incentive salience [4], but also participates in the modulation of the startle reflex [5]. The startle reflex is a rapid, involuntary, reflex muscle contraction caused by a sudden, intense stimulus [6]. One of its main features is that it is easily modulated, exhibiting different forms of plasticity, such as habituation, sensitization or prepulse modulation. Although its primary neural control occurs in mesencephalic brain structures, its plasticity is mediated by the forebrain. Therefore, it is a psychophysiological measure that reflects activation of the incentive-motivational system $[5,7]$.

Some researchers have used a cue-modulated startle test to study the appetitive response to alcohol-related signs. In this type of paradigm, the startle reflex is reliably modulated by the affective valence of foreground processing. Thus, the startle reflex is augmented while viewing unpleasant pictures and inhibited when viewing pleasant ones, compared with neutral stimuli [8]. Moreover, the eye-blink response to startling noise has also been used as an indicator of the emotional response to alcohol cues, as it is an easily quantifiable measure of behavioral reactivity to external stimuli [9].

Previous studies carried out in alcohol-dependent subjects reported an attenuation of the startle response during the presentation of alcohol-associated stimuli compared with emotionally negative and neutral stimuli. This suggests that alcohol-related stimuli may retain their appetitive qualities during alcohol abstinence $[1,2,10]$.

So far, few studies have tested the variability of the affective modulation of the cue-related startle reflex after the treatment of alcohol-dependent patients. Loeber et al. [11] showed that after a 3-week behavioral treatment based on cue exposition, intragroup changes in the magnitude of the startle reflex when viewing alcohol slides were minimal. They also demonstrated that the baseline magnitude of startle reflex elicited by alcohol-associated stimuli was a factor predicting relapse in alcohol consumption of participants after a 6-month follow-up period. However, in that study cue reactivity was assessed after the first 3 weeks of inpatient treatment, but changes occurring during the follow-up period after discharge from treatment were not assessed. Therefore, little is known about changes in the modulation of the startle reflex when patients had their alcohol-dependence treatment in an outpatient facility.

In our current study, the affective modulation of cuerelated startle reflex has been used to evaluate changes in the emotional processing of alcohol-related stimuli after a standard outpatient cognitive-behavioral intervention, and also to assess the outcome of that intervention (i.e. abstinence vs. relapse after 12 weeks of treatment, which is longer than the time lapse used by Loeber et al. [11]). One of the limitations mentioned by these authors was the difficulty of justifying such lack of significant before and after differences of startle modulation. This occurred even though treatment was efficient, which would lead to the expectation that alcohol-related stimuli would have lost their appetitive value in some way [11]. Thus, one of the objectives of the present study is to verify whether a longer treatment period would yield changes in startle modulation.

We hypothesized a 'normalization' of the startle inhibition for the alcohol-related cues during the period of treatment as a consequence of the change in salience attribution to alcohol-related stimuli. Psychophysiological reactions, such as startle reflex, are conditioned and modulated by the valence of the context or the stimulus during which they occur. Therefore, it is expected that the psychophysiological reaction to alcohol-related stimuli would have greater amplitude since, after treatment, everything related to alcohol should be considered as less appetitive. Further- 
Table 1. Clinical and demographic baseline differences among the participants

\begin{tabular}{|c|c|c|c|c|c|c|}
\hline \multirow[t]{3}{*}{ Variables } & \multicolumn{3}{|l|}{ Participants } & \multicolumn{3}{|c|}{ Significance } \\
\hline & \multicolumn{2}{|c|}{$\begin{array}{l}\text { groups of patients according to } \\
\text { their alcohol consumption during } \\
\text { the study }\end{array}$} & \multirow[t]{2}{*}{$\begin{array}{l}\text { controls } \\
(\mathrm{n}=72)\end{array}$} & \multirow[t]{2}{*}{$\mathrm{F}$} & \multirow[t]{2}{*}{ d.f. } & \multirow[t]{2}{*}{$\mathrm{p}$} \\
\hline & $\begin{array}{l}\text { abstainers } \\
(\mathrm{n}=44)\end{array}$ & $\begin{array}{l}\text { relapsers } \\
(n=28)\end{array}$ & & & & \\
\hline Years of education & $14.18 \pm 4.31$ & $15.12 \pm 11.20$ & $15.782 \pm 3.25$ & 0.94 & 1 & 0.67 \\
\hline Age of onset of alcohol use, years & $15.45 \pm 2.08$ & $16.37 \pm 2.45$ & $15.23 \pm 4.70$ & 0.83 & 1 & 0.72 \\
\hline Age of onset of alcohol dependence, years & $33.91 \pm 8.52$ & $30.21 \pm 8.80$ & - & & & \\
\hline Drinking days (previous month) & $25.30 \pm 4.18$ & $26.45 \pm 5.12$ & $16.23 \pm 6.90$ & 3.90 & 1 & 0.02 \\
\hline Standard drinks per drinking day & $19.76 \pm 7.28$ & $20.01 \pm 8.23$ & $2.11 \pm 1.34$ & 2.50 & 1 & 0.04 \\
\hline CIWA-Ar score & $4.23 \pm 2.11$ & $3.97 \pm 3.01$ & - & & & \\
\hline Negative reinforcement factor & $8.84 \pm 3.51$ & $12.39 \pm 6.36$ & & 9.302 & 1 & 0.003 \\
\hline BIS-11 score & $52.59 \pm 18.02$ & $54.00 \pm 14.04$ & $41.32 \pm 10.12$ & 2.54 & 2 & 0.04 \\
\hline
\end{tabular}

Values are given as means $\pm \mathrm{SD}$.

more, it is assumed that a higher startle inhibition at baseline would predict relapse on alcohol consumption during treatment, as it would reflect that a greater appetitive attribution to alcohol indicates greater difficulty to change.

\section{Methods}

\section{Participants}

A total of 98 alcohol-dependent subjects were included in this study. Clinical and demographic characteristics of the sample have been previously described [2]. Patients included fulfilled DSM-IV criteria for a diagnosis of alcohol dependence [12], and were determined using the validated Spanish version of the Psychiatric Research Interview for Substance and Mental Disorders (PRISM) [13]. Exclusion criteria were current drug abuse other than alcohol and nicotine, and medication that could influence psychophysiological parameters (e.g. acamprosate or naltrexone, or $\alpha$-2 adrenergic drugs such as clonidine). All participants were detoxified from alcohol 2-3 weeks before being recruited for the study. All were assessed at baseline and after 12 weeks of psychological treatment, but 26 patients dropped out of the study and only cue-reactivity measures of 72 patients were gathered after the 12 -week period. Out of these 26 patients, 10 abandoned the treatment and the clinic; therefore, their evolution is unknown. Of the remaining 16 patients, 10 exhibited artifacts in the posttreatment evaluation and were not included in the analysis (4 of them relapsed), and $6 \mathrm{did}$ not consent to repeat the tests (2 of them relapsed). Psychophysiological and clinical evaluation data of these patients were similar to those who continued the study. These baseline results were discussed in full in a previous study [2]. The final sample $(n=72)$ comprised 43 men and 29 women (mean age: $40.93 \pm 7$ years). Among them, 54.5\% were married, $8 \%$ were unemployed and $39 \%$ had a positive family history of alcoholism.

As shown in table 1, our sample was composed of patients with a mean duration of alcohol dependence of 10 years (SD 3.34) presenting a moderate severity of dependence [assessed using the Severity of Alcohol Dependence Scale (SAD)]. At baseline, $80 \%$ of patients were taking vitamin supplements $\left(B_{1}, B_{6}, B_{12}\right)$, and $60 \%$ were also receiving low doses of chlordiazepoxide (10-15 mg/ day).

The control group (43 men, 29 women, mean age: $39.47 \pm 9.24$ years) was selected to match demographic characteristics. Subjects were recruited from the community and from the staff of the mental health services. The objective of using healthy people who are not receiving any type of psychotherapy is to control the effect of time on the possible changes of the startle reflex. The inclusion of a control group of alcohol-dependent patients not included within the usual intervention programs was not considered for this study due to ethical reasons.

Before inclusion, a semistructured interview was used to screen control subjects for exclusion criteria: thyroid dysfunction, heart disease, hypotension or hypertension, regular drug prescriptions, history of mental illness, rapid mood changes, drug (determined by urine toxicology screening) and alcohol abuse, and alcohol dependence in first-degree relatives. Patients and controls differed only in variables related to alcohol use and family history of alcoholism (table 1). The study was approved by the local ethics committee. All patients signed informed consent. 


\section{Intervention}

All patients received a standard cognitive-behavioral therapy once a week throughout the study period. This manual-based group therapy oriented to relapse prevention (see characteristics of this type of therapy in Rubio et al. [14] and Alamo et al. [15]) was delivered by 2 expert psychiatrists on addictive behaviors. The cognitivebehavioral intervention was delivered weekly in a group setting (1012 patients), with a duration of $1.5 \mathrm{~h}$ per session. In these sessions, the usual topics presented in other intervention programs (dealing with situations of risk, craving and negative emotional states) were approached using psychoeducation, and the difficulties encountered were discussed by the group in the following session. These group sessions were semistructured. Abstinence was positively reinforced.

Patients also visited their psychiatrists every 15 days ( \pm 3 days) until the end of the study. In the event of relapse, the frequency of visits was increased to curtail the relapse and offer the patient assistance and to avoid abandonment and lack of assistance. Assistance frequency was similar for both groups. In each visit, the patients' entries in the alcohol diary consumption were checked. Alcohol use data were contrasted with information given by the family. During the study, disulfiram was used in 6 patients for $6-10$ days. At the endpoint assessment, 55 patients were taking vitamins and 24 patients took $5 \mathrm{mg}$ of chlordiazepoxide at some stage during the final week. One of the strategies used in general hospitals to carry out detoxification is the use of BDZ; in this case chlordiazepoxide was used. Detoxification treatment usually extends a few days longer than is strictly necessary to control the acute abstinence syndrome (7-10 days) since many patients tend to have difficulty sleeping. Chlordiazepoxide, which has a mid- to long-life action span, is reduced progressively during the detoxification period.

Patients knew that in case of anxiety or insomnia they could resort to the psychological strategies they had learned throughout their psychotherapy treatment. However, they also had permission to use chlordiazepoxide in situations they considered necessary. Thus, 24 patients admitted to taking (but only exceptionally) $5 \mathrm{mg}$ of chlordiazepoxide to manage situations of anxiety or insomnia in the last week of treatment.

\section{Assessments}

Cue reactivity was assessed before the beginning of the treatment program and at the end of the 12 -week treatment period. It included assessments of the affect-modulated startle reflex while patients were viewing alcohol-associated, as well as affective, visual stimuli. Relapse and drinking behavior of patients were assessed during the treatment period.

\section{Cue Reactivity Assessment}

Startle procedures were described in a previous study [2], but overall all participants were exposed to a set of 24 color photographs ( 6 aversive, 6 appetitive, 6 neutral and 6 alcohol-related stimuli). Eighteen images from the International Affective Picture System (IAPS) [16] were used, selected according to their valence and arousal normative values $[17,18]$. The six alcohol-related pictures depicting alcoholic beverages or typical drinking situations were used in other studies $[10,19]$. These images were standardized by Grüsser et al. [19], who developed a set of visual and olfactory stimuli specific for each substance (alcohol, tobacco, opiate and cannabis abuse for addicted patients and healthy individuals). All subjects were exposed to the same set of pictures in a random order (on the monitor). Each picture was presented for $6 \mathrm{~s}$, with a randomized interstimulus interval of 20-31 s consisting of a blank screen after picture offset. Startle probes were presented 2.5-5 s after the onset of the visual stimulus (target startle) or 4-10 s after the beginning of the interstimulus interval. All alcohol-related pictures were exhibited 4 times during the experiment and startle probes were presented in $50 \%$ of them.

The acoustic startle stimulus consisted of a $100-\mathrm{ms}$ burst of white noise at $95 \mathrm{~dB}$, with instantaneous rise time. The noise burst was presented binaurally with Sennheiser HD500 headphones (Wedemark, Germany). Each session began with four test startleprobe trials before the first picture. In addition, to enhance the unpredictability of the startle presentation, eight startle probes were presented during the interpicture intervals.

Electrodes $(\mathrm{Ag}-\mathrm{AgCl})$ filled with saline gel were attached to each participant's right orbicularis oculi region to record startle eyeblink EMG. This signal was acquired and amplified using an EMG100A module (BIOPAC Systems, Goleta, Calif., USA) connected to a MP100WSW bioamplifier (BIOPAC Systems) with a time constant of $1,000 \mathrm{~ms}$. The EMG signal was recorded at a sampling rate of $1,000 \mathrm{~Hz}$ with a low-pass filter of $500 \mathrm{~Hz}$.

The startle response represents the difference between a stable baseline (using the $50 \mathrm{~ms}$ before the startle probe) and the maximum amplitude of the startle reflex 20-150 ms after the startle probe was presented.

\section{Other Psychological and Psychiatric Assessments}

SADS [20] is derived from the SADQ (Severity of Alcohol Dependence Questionnaire). It is self-applied and includes 30 items presented in a Likert-type questionnaire format with four response alternatives ( $0-3$ points) grouped in six subscales: physical symptoms, psychological symptoms and behavior to relieve withdrawal symptoms, alcohol consumption, impaired control and reappearance of symptoms after relapses. It distinguishes between mild dependence (score $<20)$, moderate dependence (21-37) and severe dependence $(>37)$.

The Alcohol Craving Questionnaire (ACQ) [21] consists of 33 items in a Likert-type response scale with four alternatives: never, rarely, frequently and always, scoring 0, 1, 2 and 3, respectively. It is composed of three craving factors: impaired control, positive reinforcement and negative reinforcement. The period referred in the questionnaire comprises the few weeks of consumption prior to detoxification treatment.

Patients' reports of symptom severity were rated using the revised Clinical Institute of Alcohol Withdrawal Scale (CIWA-Ar) [22].

The Barratt Impulsiveness Scale, version 11 (BIS-11) is a selfreport multidimensional measure of impulsivity consisting of 30 items and cognitive, motor, and non-planning subscales [23].

\section{Assessment of Alcohol Consumption}

In the post-treatment period, we used the 'Timeline Followback' interview during the psychiatrist interview every 2 weeks. We assessed 'relapse' by a yes-no variable defined as any consumption of ethanol.

\section{Statistical Analyses}

The main aim of this study was to investigate if the affective modulation of cue-related startle reflex might be useful to assess the outcome of a standard cognitive-behavioral intervention and the emotional processing of alcohol-associated stimuli. In order to attain such goals, the following strategy was adopted. 
Table 2. Startle probe magnitudes for alcohol-dependent patients at baseline and after the 12 -week treatment period

\begin{tabular}{|c|c|c|c|c|c|c|}
\hline \multirow[t]{2}{*}{ Startle magnitudes } & \multicolumn{2}{|c|}{$\begin{array}{l}\text { Alcohol-dependent patients } \\
\text { abstainers }(\mathrm{n}=44)\end{array}$} & \multicolumn{2}{|c|}{$\begin{array}{l}\text { Alcohol-dependent patients } \\
\text { relapsers }(n=28)\end{array}$} & \multicolumn{2}{|c|}{ Controls $(n=72)$} \\
\hline & baseline & after treatment & baseline & after treatment & baseline & after treatment \\
\hline Alcohol startle magnitude & $48.12(4.71)^{\mathrm{a}}$ & $50.58(4.11)^{\mathrm{e}}$ & $46.04(3.82)$ & $51.06(5.41)$ & $53.03(14.12)$ & $53.73(10.33)$ \\
\hline Appetitive startle magnitude & $53.22(8.13)^{\mathrm{b}}$ & $51.46(8.44)^{\mathrm{f}}$ & $51.48(7.58)$ & $50.09(7.87)$ & $51.02(12.08)$ & $51.78(10.20)$ \\
\hline Neutral startle magnitude & $56.34(4.87)^{\mathrm{c}}$ & $57.58(8.11)^{\mathrm{g}}$ & $55.87(4.77)$ & $56.87(9.89)$ & $54.12(12.11)$ & $54.73(14.70)$ \\
\hline
\end{tabular}

Startle magnitudes are expressed in $\mu \mathrm{V}$. Values are given as means (SD). Differences among groups at baseline: ${ }^{a}$ relapsers $<$ abstainers $<$ controls; ${ }^{\mathrm{b}}$ relapsers $=$ abstainers $=$ controls; ${ }^{\mathrm{c}}$ relapsers $=$ abstainers $=$ controls; ${ }^{\mathrm{d}}$ relapsers $<$ controls $<$ abstainers. Differences among groups at 12 weeks: ${ }^{\mathrm{e}}$ relapsers $=$ abstainers $<$ controls; ${ }^{\mathrm{f}}$ relapsers $=$ abstainers $=$ controls; ${ }^{\mathrm{g}}$ relapsers $=$ abstainers $>$ controls; $\mathrm{h}$ abstainers $>$ controls $>$ relapsers.

First, $60 \%$ of patients were using low doses of chlordiazepoxide at baseline (preintervention) startle reflex evaluation. Since this medication might bias a patient's physiological response, a preliminary analysis was performed to evaluate its potential influence. Patients were split into two subgroups and medication effects on startle reflex were evaluated by $2 \times 4$ repeated-measures mixed-effects analysis of variance (ANOVA). The between-subjects factor was 'group' (medicated vs. nonmedicated), and the within-subjects repeated measures was 'type of stimuli' (appetitive, aversive, alcohol-associated, and neutral). In cases of statistical significance, the post hoc pairwise means comparison was carried out using Bonferroni correction for multiple comparisons.

Once this exploratory analysis was performed, $3 \times 2 \times 4$ repeated-measures mixed-effects ANOVA was done to evaluate intervention and outcome influences on cue-related startle reflex. The between-subjects factor was 'outcome' (abstinence, relapse and control; see below), the within-subjects repeated measures were 'intervention' (preintervention vs. postintervention) and 'type of stimuli' (appetitive, aversive, alcohol-associated and neutral). Again, in cases of statistical significance, the post hoc pairwise means comparison was carried out using Bonferroni correction for multiple comparisons.

Finally, we developed a logistic regression model to predict patients' outcome (abstinence vs. relapse). The predictive capability of baseline cue reactivity produced by affective and alcohol-associated stimuli was investigated. Factors with the highest predictive capability were selected. The model's performance was assessed by means of correct classification percentages, together with the usual goodness-of-fit tests.

All statistical analyses were performed using SPSS $19^{\circledR}$.

\section{Results}

\section{Preliminary Analysis of Medication Effects}

Cue-related startle reflex was not influenced by the main effect of 'group' (medicated vs. nonmedicated; $\left.\mathrm{F}_{1,70}=0.002 ; \mathrm{p}=0.927\right)$ or by the interaction of this vari- able with 'type of stimuli' $\left(\mathrm{F}_{3,210}=0.155 ; \mathrm{p}=0.964\right)$. According to these results, we might claim that baseline startle reflex was not affected by medication effects, and consequently we did not include this factor in the ANOVA presented in the following section.

\section{Patient Group}

During the treatment period, 28 patients $(39 \%)$ relapsed in the consumption of alcohol, out of which 17 were women. Table 1 shows baseline differences among patients who remained abstinent for 12 weeks (abstainer group, $\mathrm{n}=44$ ), those who relapsed (relapser group, $\mathrm{n}=$ 28) and controls. Out of these 28 patients, 15 relapsed during the first month of treatment, 8 during the second month and 5 during the third month. The average duration of relapse was 1.78 days (SD 2.8).

With respect to patients, at baseline, relapsers had significantly higher scores on craving subscales than abstainers (table 1). The former group also exhibited more startle inhibition when viewing alcohol-related $(\mathrm{p}=$ $0.034)$ and aversive scenes $(p=0.016)$ than abstainers. According to these results, the patient group was split into two subgroups (relapsers vs. abstainers) for further statistical analyses.

Outcome and Intervention (before vs. after) Influences on Cue-Related Startle Reflex

Cue-related startle reflex was modified by the significant main effects of the 'outcome' $\left(\mathrm{F}_{2,141}=3.956\right.$; $\left.\mathrm{p}<0.05\right)$ and 'type of stimuli' $\left(\mathrm{F}_{3,423}=53.056 ; \mathrm{p}<0.001\right)$ factors. These effects were confirmed by the significant interaction of outcome $\times$ type of stimuli $\left(\mathrm{F}_{6,423}=6.616 ; \mathrm{p}<\right.$ 0.001 ), and by the significant triple interaction of outcome $\times$ intervention $\times$ type of stimuli $\left(\mathrm{F}_{6,423}=3.485 ; \mathrm{p}<\right.$ 
Fig. 1. Startle magnitude in all conditions (before and after treatment). ${ }^{*} \mathrm{p}<0.05$; ** $\mathrm{p}<0.01$.

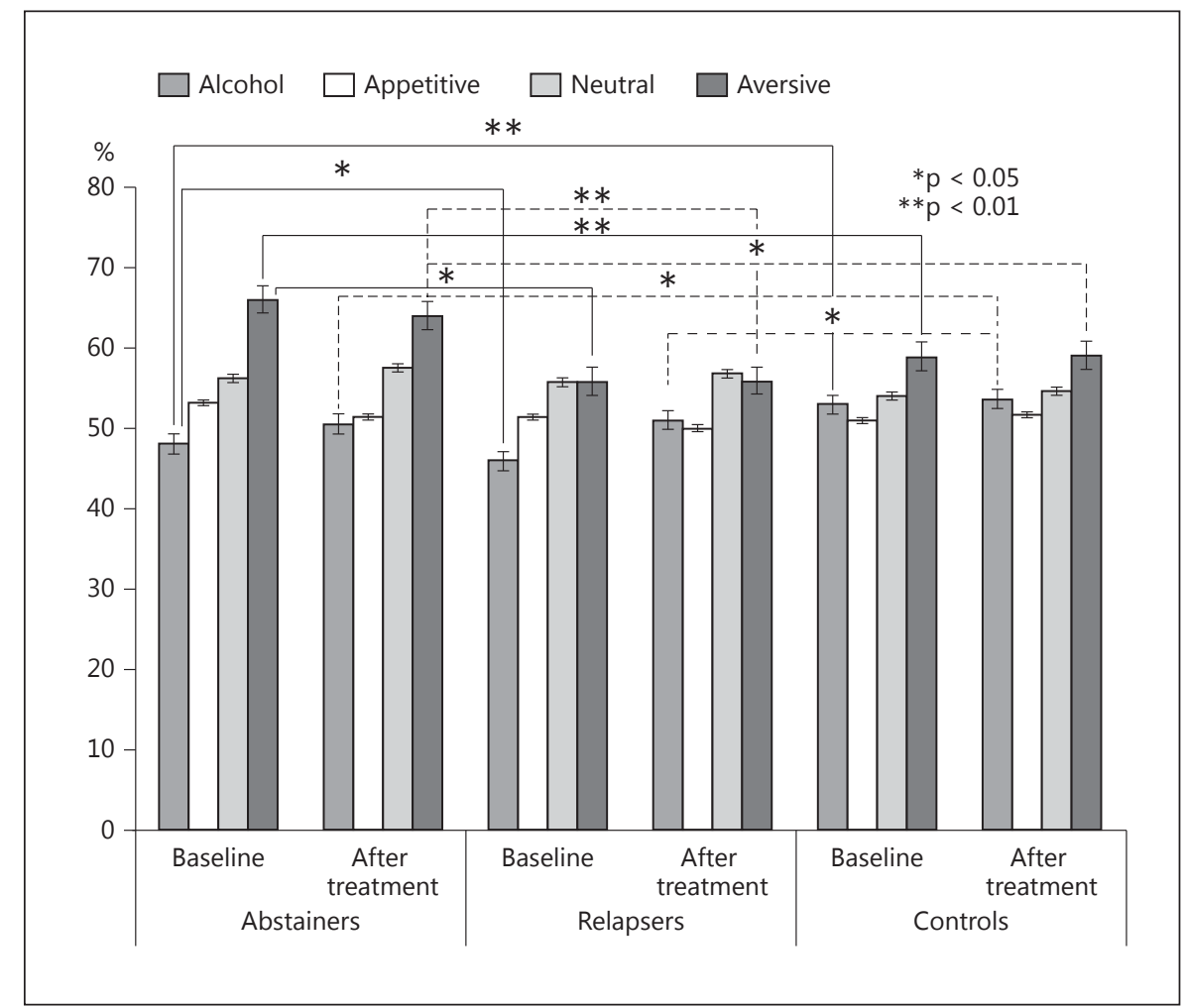

0.05; table 2). In patients at baseline as well as after the 12 -week treatment period, the startle reflex was higher for aversive cues, followed by neutral, appetitive and alcoholrelated cues. This pattern also occurred in the control group, except for the startle reflex in alcohol cues, which was between the startle reflex for neutral cues and appetitive cues.

The emotional modulation effects were modified by the 'outcome' and 'intervention' factors. Alcohol-related and aversive stimuli produced a significantly lower startle reflex within the relapser group compared with abstainers and controls at the preintervention evaluation (Bonferroni correction for multiple comparisons, alcohol related: relapsers $<$ abstainers, $\mathrm{p}=0.034$; abstainers $<$ control, $\mathrm{p}=$ 0.034 ; aversive: abstainers $>$ relapsers, $\mathrm{p}=0.019$; abstainers $>$ controls, $p=0.004$ ). Additionally, intervention produced a different evolution of the startle reflex in relapsers and abstainers (fig. 1). Postintervention measures of startle reflex elicited by aversive stimuli were significantly higher for the abstinence group, followed by controls and relapsers (Bonferroni correction for multiple comparisons, aversive: abstainers $>$ control, $\mathrm{p}=0.036$; $a b-$ stainers $>$ relapsers, $\mathrm{p}=0.009$ ). The startle response to alcohol pictures increased in both patient groups in con- trast to the control group after the 12 -week treatment period (Bonferroni correction for multiple comparisons, abstainers: baseline < posttreatment, $\mathrm{p}=0.012$; relapsers: baseline $<$ posttreatment, $\mathrm{p}=0.000$ ), although that response was still significantly lower in patients. The evolution of the startle reflex to aversive- and appetitive-related cues were different to the alcohol-related cues. After the 12 -week treatment period, the startle response to aversive-related cues decreased in the abstinence group (Bonferroni correction for multiple comparisons, baseline > posttreatment, $p=0.014$ ) and there were no significant changes in relapsers or controls. The relapse group continued to have the lowest response compared to the other groups. Related to the appetitive response, the startle response decreased in abstainers (Bonferroni correction for multiple comparisons, baseline $>$ posttreatment, $\mathrm{p}=$ 0.000 ) and there were no significant modifications in the other two groups (fig. 1).

\section{Prediction of Intervention Outcome}

The variable selection process began with a careful univariate analysis of each variable, using the likelihood ratio test as selection criteria. As mentioned above, the variables included in this analysis were the scores of 
SADS, ACQ and BIS-11, together with baseline startle responses elicited by aversive, appetitive, neutral and alcohol-associated stimuli. Forward stepwise regression was used to build the final model. In this case, only one of the variables, the preintervention startle reflex elicited by alcohol-associated stimuli, was statistically significant ( $\mathrm{p}=$ 0.018 ) in the univariate and multivariate analyses. The Nagelkerke $\mathrm{R}^{2}$ goodness-of-fit statistic (NS) was 0.116 , and the goodness of fit in $-2 \log$ likelihood units was 88.91 .

The fitted univariate model had the following coefficients: constant $\mathrm{B}_{0}=6.372, \mathrm{~B}_{\text {alcohol-associated }}=-13.381$. This model indicated that lower values of the baseline startle reflex elicited by alcohol-associated stimuli were associated with a higher probability of being included in the relapse group. Finally, the percentage of correct classification of the logistic regression model was $75 \%$ for the abstinence group and $53.6 \%$ for the relapse group.

\section{Discussion}

As far as we know, there are few studies in which changes in affective modulation of startle reflex elicited by alcohol stimuli have been assessed in alcohol-dependent patients who received a standard cognitive-behavioral therapy. The most relevant findings of our study were that the startle response predicted relapse. It also differed significantly after 12 weeks of treatment for alcohol-related, neutral and aversive stimuli between alcoholdependent patients and controls: at the postintervention evaluation, abstainers and relapsers still exhibited lower startle magnitudes for alcohol-associated stimuli than controls (meaning that the appetite for alcohol is still higher in patients than in controls). At baseline, subjects who relapsed during the treatment period exhibited lower startle reflex when viewing alcohol-related images (these were processed as more appetitive) and also when they were exposed to aversive pictures (these were processed with less aversion than the nonrelapse group). Thus, in our study, lower values of the baseline startle reflex elicited by alcohol-associated stimuli were associated with higher probabilities of relapse on alcohol use.

After treatment, both groups of patients showed a slight increase in the amplitude of the startle reflex to alcohol-related stimuli, suggesting that indeed, after 12 weeks of intervention, alcohol is considered less appetitive. However, the amplitude is still lower than for the control group. This could be related to a change in the motivational relevance of such stimuli, which could be the beginning of the extinction of conditioning. A more long-term assessment and a control group of alcoholic patients not receiving any cognitive-behavioral treatment would be required to verify whether this change is clinically and statistically relevant and due to treatment.

Therefore, our results may indicate that the startle reflex refers to more enduring and permanent processes of cue reactivity and that the emotional processing of alcohol-associated cues assessed with the affect-modulated startle reflex is less affected by interventions attempting to influence explicit cognitions than previously assumed [11].

The startle magnitude when viewing alcohol-related stimuli has been considered as an 'objective measure of alcohol craving' [10] and has also been related to alcoholdependence severity $[2,10]$. Two categories of drug reactions are distinguished in subjects with alcohol dependence: alcohol-like and alcohol-opposite effects [24]. Stimuli linked to alcohol may evoke a positive affective condition or focus attention on reward, indicating stimuli and thus lead to the renewed search for the drug [25]. However, an aversive physical and psychological condition caused primarily by conditioned withdrawal symptoms may precede craving and alcohol intake [26]. The different reactions to alcohol-associated stimuli may be mediated by different neurobiological pathways $[27,28]$. From this perspective, our study supports the hypothesis that in late abstinence, visual alcohol stimuli evoke a persistent and positive basic motivational state that extends at least for 12 weeks after treatment [2]. Our data are in line with an incentive-motivational model of drug cues [3], and also suggest that the sensitization towards alcohol cues is the neurobiological correlate of an 'addiction memory' and may represent a process highly resistant to overwriting $[10,25,29]$.

Several studies have suggested that increased alcohol expectancies predict decreased reactivity to salient stimuli, particularly those of an aversive nature [30-32]. The fact that relapsers had, at baseline, lower startle reflex than abstainers when they were exposed to aversive stimuli may suggest that this group considers the stimuli presented as less aversive. This data may support the idea that patients who relapse have a greater alteration in their incentivemotivational system, making a pathological salience attribution. Thus, this group does not consider relevant stimuli that are usually considered as relevant [33]. The fact that stimuli not associated with consumption are attributed an inadequate relevance, together with a deficit in inhibitory control, can result in a misguided decision-making process which yields inadequate behaviors towards consumption 
or relapse in our sample, without considering the longterm negative consequences $[4,33,34]$.

This alteration in the regulation of affective response has been demonstrated in several studies, with different psychophysiological measures. Moreover, the hyporesponse to aversive stimuli seems to be an explicit feature of subjects at risk (with family history of alcoholism) or of specific subgroups of alcoholics who have antisocial personality traits [30-32]. Miranda et al. [31, 32] evaluated, in both types of samples, the affective modulation of the startle reflex. Subjects were exposed to pleasant, neutral and unpleasant pictures, but not to alcohol-related pictures. Findings from these studies have shown that the typical potentiation of EMG with unpleasant stimuli was not observed in young adults with a family history of alcoholism [31] or with antisocial personality disorder [32].

Among startle reflex variables recorded at baseline, a lower startle magnitude for alcohol cues was associated with a higher probability of relapse during the 12 weeks of treatment. Similar results have been reported by Loeber et al. [11]. Lower startle magnitude for alcohol-related stimuli has been associated with severity of alcohol dependence $[2,10]$ and with family history of alcoholism $[2$, 31]. Furthermore, those variables have been associated with poor outcome in alcohol dependence $[14,35]$. From this view, the startle reflex magnitude for alcohol-associated stimuli could be considered as a psychophysiological marker of alcoholism severity, in line with studies showing increased activation elicited by alcohol cues in the prefrontal cortex predicted relapse [36].

Our study has some limitations that should be considered for future research. The evaluated period includes only the treatment period - it would be desirable to obtain long-term measures to check if the startle reflex modulation is related to the patients' evolution and abstinence maintenance once the treatment period is completed. In addition, the obtained results could be more solid if patients who were not undergoing any type of cognitive behavioral therapy had been included in the study. Thus, conclusions could be drawn about the efficacy of cognitive intervention itself. However, we believe that not allowing a group of patients to be included into the treatment program is unethical. On the other hand, those patients who did not accept to be included in the conventional treatment also declined to participate in our study.

Further studies are necessary to demonstrate the stability of the appetitive nature of drug-associated cues and to find further evidence for the predictive value of the startle reflex. At present, our results show that the attenuation of the startle reflex when patients viewed alcohol-

related and aversive slides were significant predictors of relapse during treatment. These results stress the role of the startle reflex as an indicator of processes underlying drinking behavior.

There are several clinical implications of our study. The fact that we found no significant changes in the starthe reflex responses to alcohol stimuli after 12 weeks suggests that psychological or pharmacological treatments for relapse prevention of alcohol-dependent subjects may have to be extended for periods longer than 3 months. Furthermore, taking our results into consideration, it seems clear that the attribution of motivational relevance is a clear predictor of relapse. Therefore, prevention programs should make a greater effort to get patients to return to a normal relevance attribution system, in which natural stimuli, both positive and negative, have an appropriate saliency. Hence, it would be advisable to include, along with the usual cognitive-behavioral techniques (relaxation, relapse prevention, thought stopping, social skills, contingency management, etc.), specific techniques aimed at identifying and controlling negative emotions.

\section{Acknowledgments}

This research was supported by grant PI041989 (Fondo de Investigaciones Sanitarias) and by Instituto de Salud Carlos III (RETICS, RD06/0001/1004). S.M. was financed by a Ramón y Cajal fellowship.

\section{Disclosure Statement}

All authors reported no biomedical financial interests or potential conflicts of interest.

References

Eur Addict Res 2015;21:195-203 DOI: $10.1159 / 000371723$
1 Mucha RF, Geier A, Stuhlinger M, Mundle G: Appetitve effects of drug cues modelled by pictures of the intake ritual: generality of cuemodulated startle examined with inpatient alcoholics. Psychopharmacology (Berl) 2000; 151:428-432.

2 Rubio G, Borrell J, Jimenez M, Jurado R, Grusser SM, Heinz A: Variables involved in the cue modulation of the startle reflex in alcohol-dependent patients. Addict Biol 2013; 18:170-180.

3 Kelley AE, Berridge KC: The neuroscience of natural rewards: relevance to addictive drugs. J Neurosci 2002;22:3306-3311.

4 Robinson TE, Berridge KC: Addiction. Annu Rev Psychol 2003;54:25-53. 
5 Bradley M, Sabatinelli D: Startle reflex modulation: perception, attention and emotion; in Hugdahl K (ed): Experimental Methods in Neuropsychology. Waltham, Kluwer Academic Publishers, 2003, pp 65-88.

6 Wilkins D, Hallett M, Wess M: Audiogenic startle reflex of man and its relationship to startle syndromes. Brain 1986;109:561-573.

7 Swerdlow NR, Caine SB, Braff DL, Geyer MA: The neural substrates of sensorimotor gating of the startle reflex: a review of recent findings and their implications. J Psychopharmacol 1992;6:176-190.

8 Bradley MM, Cuthbert BN, Lang PJ: Pictures as prepulse: attention and emotion in startle modification. Psychophysiology 1993;30: 541-545.

9 Morgan CA 3rd, Southwick SM, Grillon C, Davis M, Krystal JH, Charney DS: Yohimbine-facilitated acoustic startle reflex in humans. Psychopharmacology (Berl) 1993;110: 342-346.

10 Grüsser SM, Heinz A, Raabe A, Wessa M, Podschus J, Flor H: Stimulus-induced craving and startle potentiation in abstinent alcoholics and controls. Eur Psychiatry 2002;17:188193.

11 Loeber S, Croissant B, Nakovics H, Zimmer A, Georgi A, Klein S, et al: The startle reflex in alcohol-dependent patients: changes after cognitive-behavioral therapy and predictive validity for drinking behavior. A pilot study. Psychother Psychosom 2007;76:385-390.

12 Diagnostic and Statistical Manual of Mental Disorders, ed 4, text revision. Arlington, American Psychiatric Association, 2000.

13 Torrens M, Serrano D, Astals M, PerezDominguez G, Martin-Santos R: Diagnosing comorbid psychiatric disorders in substance abusers: validity of the Spanish versions of the Psychiatric Research Interview for Substance and Mental Disorders and the Structured Clinical Interview for DSM-IV. Am J Psychiatry 2004;161:1231-1237.

14 Rubio G, Jimenez-Arriero MA, Ponce G, Palomo T: Naltrexone versus acamprosate: one year follow-up of alcohol dependence treatment. Alcohol Alcohol 2001;36:419-425.
15 Alamo C, López-Muñoz F, Rubio G: Avances en Drogodependencias. Las Palmas de Gran Canaria, GAFOS, 2008.

16 Lang PJ, Bradley MM, Cuthbert BN (ed): International Affective Picture System (IAPS). Affective ratings of pictures and instruction manual. International Affective Picture System (IAPS). Affective Ratings of Pictures and Instruction Manual. Gainesville, Fla., University of Florida, 2005.

17 Moltó J MS, Poy R, Segarra P, Partor MC, Tormo MP: Un nuevo método para el estudio experimental de las emociones: El International Affective Picture System (IAPS). Adaptación española. Revista de Psicología Aplicada, 1999;52:55-87.

18 Vila J, Sánchez M, Ramirez I, Fernández MC, Cobos P, Rodriguez S: El Sistema Internacional de Imágenes Afectivas (IAPS). Adaptación española. Revista de Psicología Aplicada 2001;54:635-657.

19 Grüsser SM, Heinz A, Flor H: Standardized stimuli to assess drug craving and drug memory in addicts. J Neural Transm 2000;107: 715-720.

20 Rubio G, Urosa B, Santo-Domingo J: Validación de la escala de la intensidad de la dependencia alcohólica (EIDA). Psiquiatría Biológica 1998;(suppl 1):44-47.

21 Jimenez M, Graña JL, Montes V, Rubio G: Alcohol craving scale based on three factors. Eur Addict Res 2009;15:135-142.

22 Sullivan JT, Sykora K, Schneiderman J, Naranjo CA, Sellers EM: Assessment of alcohol withdrawal: the revised clinical institute withdrawal assessment for alcohol scale (CIWAAr). Br J Addict 1989;84:1353-1357.

23 Patton JH, Stanford MS, Barratt ES: Factor structure of the Barratt impulsiveness scale. J Clin Psychol 1995;51:768-774.

24 O'Brien CP, Childress AR, McLellan AT, Ehrman R: A learning model of addiction. Res Publ Assoc Res Nerv Ment Dis 1992;70:157177.

25 Robinson TE, Berridge KC: The neural basis of drug craving: an incentive-sensitization theory of addiction. Brain Res Brain Res Rev 1993; 18:247-291.

26 Siegel S: Drug anticipation and drug addiction. The 1998 H. David Archibald Lecture. Addiction 1999;94:1113-1124.
27 Verheul R, van den Brink W, Geerlings P: A three-pathway psychobiological model of craving for alcohol. Alcohol Alcohol 1999;34: 197-222.

28 Heinz A, Lober S, Georgi A, Wrase J, Hermann D, Rey ER, et al: Reward craving and withdrawal relief craving: assessment of different motivational pathways to alcohol intake. Alcohol Alcohol 2003;38:35-39.

29 Koob GF: Dynamics of neuronal circuits in addiction: reward, antireward, and emotional memory. Pharmacopsychiatry 2009;42(suppl 1):S32-S41.

30 Drobes DJ, Carter AC, Goldman MS: Alcohol expectancies and reactivity to alcohol-related and affective cues. Exp Clin Psychopharmacol 2009;17:1-9.

31 Miranda R Jr, Meyerson LA, Buchanan TW, Lovallo WR: Altered emotion-modulated startle in young adults with a family history of alcoholism. Alcohol Clin Exp Res 2002;26: 441-448.

32 Miranda R Jr, Meyerson LA, Myers RR, Lovallo WR: Altered affective modulation of the startle reflex in alcoholics with antisocial personality disorder. Alcohol Clin Exp Res 2003;27:1901-1911.

33 Verdejo-Garcia A, Perez-Garcia M, Bechara A: Emotion, decision-making and substance dependence: a somatic-marker model of addiction. Curr Neuropharmacol 2006;4:17-31.

34 Verdejo-Garcia A, Bechara A, Recknor EC, Perez-Garcia M: Negative emotion-driven impulsivity predicts substance dependence problems. Drug Alcohol Depend 2007;91: 213-219.

35 Kiefer F, Jimenez-Arriero MA, Klein O, Diehl A, Rubio G: Cloninger's typology and treatment outcome in alcohol-dependent subjects during pharmacotherapy with naltrexone. Addict Biol 2008;13:124-129.

36 Beck A, Wustenberg T, Genauck A, Wrase J, Schlagenhauf F, Smolka MN, et al: Effect of brain structure, brain function, and brain connectivity on relapse in alcohol-dependent patients. Arch Gen Psychiatry 2012;69:842852. 